\title{
Predicting complete cytoreduction for advanced ovarian cancer patients using nearest-neighbor models
}

\author{
Alexandros Laios ${ }^{1 *} \mathbb{D}$, Alexandros Gryparis², Diederick DeJong ${ }^{1}$, Richard Hutson ${ }^{1}$, Georgios Theophilou ${ }^{1}$ and \\ Chris Leach L $^{3,4}$
}

\begin{abstract}
Background: The foundation of modern ovarian cancer care is cytoreductive surgery to remove all macroscopic disease (RO). Identification of RO resection patients may help individualise treatment. Machine learning and Al have been shown to be effective systems for classification and prediction. For a disease as heterogenous as ovarian cancer, they could potentially outperform conventional predictive algorithms for routine clinical use. We investigated the performance of an Al system, the k-nearest neighbor ( $k$-NN) classifier, to predict R0, comparing it with logistic regression. Patients diagnosed with advanced stage, high grade serous ovarian, tubal and primary peritoneal cancer, undergoing surgical cytoreduction from 2015 to 2019, was selected from the ovarian database. Performance variables included age, BMI, Charlson Comorbidity Index, timing of surgery, surgical complexity and disease score. The $k$-NN algorithm classified RO vs non-RO patients using 3-20 nearest neighbors. Prediction accuracy was estimated as percentage of observations in the training set correctly classified.
\end{abstract}

Results: 154 patients were identified, with mean age of $64.4 \pm 10.5 \mathrm{yrs}$., BMI of $27.2 \pm 5.8$ and mean SCS of $3 \pm 1$ (1-8). Complete and optimal cytoreduction was achieved in $\overline{2}$ and $88 \%$ patients. The mean predictive accuracy was $66 \%$. R0 resection prediction of true negatives was as high as $90 \%$ using $k=20$ neighbors.

Conclusions: The $k$-NN algorithm is a promising and versatile tool for RO resection prediction. It slightly outperforms logistic regression and is expected to improve accuracy with data expansion.

Keywords: Ovarian Cancer, Cytoreduction, Predictive factors, Machine learning, Artificial intelligence

\section{Background}

Ovarian, tubal and primary peritoneal cancer is the most lethal malignancy in women with 5-year survival not exceeding $30 \%$ [1]. The epithelial ovarian cancer (EOC) is the most frequent type representing $90 \%$ of all cases. Up to $60 \%$ of these cancers are diagnosed at an advanced stage (International Federation of Gynaecology and Obstetrics stage III and IV aEOC). Standard therapy

\footnotetext{
* Correspondence: alxlaios2000@yahoo.com

'Department of Gynaecological Oncology, St James's University Hospital, Leeds Teaching Hospitals, Leeds LS9 7TF, UK

Full list of author information is available at the end of the article
}

comprises a combination of cytoreductive surgery and platinum-based chemotherapy, either as treatment following surgery (adjuvant) or as treatment both before and after surgery (neoadjuvant, NACT) [2]. Complete Cytoreduction (CCR) to no macroscopically visible residual disease (R0) is the mainstay of primary treatment. Cytoreductive outcome and tumor load are the most significant modifiable markers of survival [3, 4]. Following recent publications of landmark randomised studies demonstrating non-inferiority of NACT over primary surgery, it appears that NACT achieves higher R0 rates but, paradoxically, the survival rates are comparable [4]. 
To achieve macroscopic tumor clearance in peritoneally disseminated disease, maximal surgical effort is required, potentially including multi-visceral resection techniques, resulting in improved rates of cytoreduction [5].

Development of methods to predict surgical outcomes in addition to prognosis is an important paradigm in the realm of personalized medicine [6]. Optimal cytoreductive surgery for aEOC is pivotal for improving overall survival and disease-free survival. Developing methods to predict resectability of the disease will identify those who will benefit from maximal cytoreductive effort in a primary or interval surgical setting. Therefore, the risk of false negatives requires a final assessment of resectability as the first stage of cytoreductive surgery by laparotomy. Numerous composite models, including several aspects of preoperative work up and, sometimes, laparoscopy have been proposed to improve the accuracy of the predictive process [7].

Artificial intelligence (AI) has proven to have an enormous potential in many areas of healthcare with the added benefits of handling enormous amounts of biomedical data, coping with missing data and evolving in the presence of new data [8]. Machine learning (ML) is a branch of AI technology that allows computers to "learn" potential patterns from past examples. This approach has been used to predict cancer survival or optimal cancer drug therapies $[9,10]$.

Nevertheless, for a disease as heterogenous as ovarian cancer, accurate prediction is difficult with conventional statistics because patient characteristics show a multidimensional and non-linear relationship. There is evidence that ML methods can perform better in such setting than traditional statistical methods, familiar to clinicians, in handling complex information derived from large datasets with multiple input variables [11].

We aimed to investigate the performance of an ML system, the $k$-nearest neighbor $(k-\mathrm{NN})$ classifier to predict $\mathrm{R} 0$ in high grade serous aEOC patients and compare it with conventional logistic regression. We hypothesised that certain predictors for R0 may work best for restricted subsets of these patients resulting in improved prediction accuracy. Therefore, the $k$-NN approach would identify those previously treated patients who most closely match the target patient (hence "nearest neighbors") on intake variables. This strategy has been used to estimate the probabilities of alpine avalanches occurring [12]. It has been also used to predict treatment response to psychotherapy [13].

\section{Methods}

We reviewed all patients diagnosed with histologically proven high grade serous only aEOC between Jan 2015 and Dec 2019 who were considered for cytoreductive surgery as part of their treatment pathway. All patients were managed at the Leeds Teaching Hospital Trust, Leeds, UK, which has been recently accredited by ESGO as a Centre of Excellence for ovarian cancer surgery. All patients were discussed at the central multi-disciplinary team (MDT) meeting and prospectively recorded in an electronic database. Patients were considered for cytoreductive surgery if the initial diagnostic workup (including physical examinations, hematological-biochemical examinations, tumor biomarkers, and CT scans) suggested a successful cytoreduction was feasible. Women either underwent primary debulking surgery (PDS) or 3-4 cycles NACT followed by interval debulking surgery (IDS) if: stage 4 disease; poor performance status; uncertainty about the possibility of optimal tumor removal. Women not exposed to surgery - those with progressive disease despite NACT, worsening performance status, and patient choice - were excluded. This retrospective observational cohort study was approved by the ethics review board.

K-NN models were applied to classify R0 vs non-R0 patients. In the $k$-NN approach, the intake variables are used only to identify the nearest neighbors for each specific subject, i.e. those subjects with intake variable values similar to the specific subject. Individual change is then predicted employing an unconditional growth model, using the average growth for the NNs as the prediction. Then, the optimum number of neighbors $k$ was estimated based on the error calculation of the validation test. The higher $k$, the smoother the decision boundaries become. As $k$ increases, we may end up in overfitting.

Specifically, we investigated models using three to 20 nearest neighbors. From the 147 patients who provided all observations in all required variables, we set 96 randomly chosen patients for the training set and the remaining 51 for the test set (i.e. a ratio of 65/35), and we repeated the process 500 times [14]. We compared the performance of $k-\mathrm{NN}$ models with that of multiple logistic regression using the same predictors in both approaches. For each iteration we estimated the prediction accuracy as the percentage of observations in the validation set that was correctly classified in each approach. Furthermore, for each iteration we estimated the percentage of true positives (i.e. patients with R0 correctly classified), true negatives (i.e. patients without R0 correctly classified), false positives and false negatives. In terms of predictors, for both approaches we used age, BMI, type of surgery, Charlson Comorbidity Index (CCI), Surgical Complexity Score (SCS), preTxCA125 and disease score. SCS was assigned based on the Aletti classification as low, intermediate and high. All quantitative variables were normalized before applying the $k$ - $\mathrm{NN}$ models.

Categorical variables were presented as absolute and relative (\%) frequencies. Continuous variables were 
presented using appropriate descriptive statistics (i.e. mean, median, SD, min, max). Quantitative variables were compared in R0 vs non-R0 patients using MannWhitney tests, while the association between R0 and non-R0 patients with qualitative variables was investigated using chi-square tests. All analyses were implemented in $\mathrm{R}$ statistical software using the library class and the knn() function (R Core Team (2017) [15] and IBM SPSS v. 25 (IBM Corp. Released 2017) [16].

\section{Results}

A total of 154 high grade serous aEOC patients receiving treatment at Leeds Cancer Center were identified. Mean age and BMI were $64.4 \pm 10.5$ yrs. and $27.2 \pm 5.8$ respectively. The mean SCS was $3 \pm 1$ (1-8). Of these patients, $31 / 154(20 \%)$ underwent primary and 123/154 (80\%) interval cytoreduction, respectively. Complete and optimal cytoreduction was achieved in 96/154 (62.3\%) and $135 / 154(87.7 \%)$ patients. The patients' characteristics are summarised in Tables 1 and 2 . From the variables selected to predict $\mathrm{R} 0$ resection, only disease score was significantly different between $\mathrm{R} 0$ and non-R0 patients $(p=0.0006)$.

To predict R0 resection, the $k$-NN model was employed to classify patients in R0 versus non-Ro resection (Fig. 1). The classifier uses one tuning parameter $(k)$ and is sensitive to data sampling and the number of neighbors $k$.

The predictive accuracy of the model for different choices of $k$ is shown in Table 3. The highest mean predictive accuracy for $k$-NN methods was $65.8 \%$ (achieved for $k=15$ and $k=19$ ). The minimum predictive accuracy showed the lower bound of performance and hence the level of difficulty on the prediction problem in hand. The lowest performance bound was around $40 \%(k=4)$ but the maximum predictive accuracy became as high as $82.4 \%$ for $\mathrm{k}=12$ and $\mathrm{k}=15$. Nevertheless, the $k$-NN model performed slightly better than logistic regression for the selected number of neighbors $k$ (Table 3 ).

In addition to performance comparison, we analysed the most important variables that contributed to the prediction model (R0 resection). The relative importance of selected clinical variables was quantified by calculating the prediction accuracy/error rate in relation to the number of predictors included in the models. Figure 2 displays the misclassification error of selected predictors in the training set for a given neighborhood size. For $k=$ 15 , $\mathrm{R} 0$ resection is best predicted by a $k \mathrm{NN}$ model that includes age and disease score only. Notably, if surgical complexity score is added, the model performs worse with R0 resection prediction accuracy just above $78 \%$.

Table 4 demonstrates a prediction comparison for CCR between the $k \mathrm{NN}$ model and logisitic regression on a random sample of 20 patients from the training set, who underwent R0 resection. It also provides information on the potential clinical use of the predictive $\mathrm{NN}$ strategy to counsel individual patients about their surgery. Patient 2 was a 66-year-old woman, slightly overweight, who returned to outpatients for discussion about interval debulking surgery. She had a history of ischaemic heart disease. Her pretreatment CA125 was $466 \mathrm{u} /$ $\mathrm{mL}$. She had a good partial response to chemotherapy and on imaging, her disease appeared limited to the pelvis. In her case, complete cytoreduction could be predicted from two to four previously treated patients with similar BM, limited or no co-morbidities, who underwent interval cytoreduction. Selecting information from more previously treated patients of the same age group would aid R0 resection prediction even if a standard cytoreductive surgery was offered. (Table 4).

\section{Discussion}

In EOC, it is widely accepted that R0 resection following cytoreductive surgery is associated with the best overall outcomes [17]. For a disease as heterogenous as EOC, a standard "one-size fits all" approach for surgical cytoreduction cannot be acceptable. Predicting surgical outcomes and stratifying patients with respect to CCR based on clinical information is fundamental towards individualised optimal cancer care. This study describes the development of an ML model, which can be directly

Table 1 Descriptive statistics for the continuous variables, by group and overall

\begin{tabular}{|c|c|c|c|c|c|c|c|c|}
\hline R0 vs non Ro & & $\mathrm{N}$ & Mean & Median & SD & Min & Max & $P$ \\
\hline \multirow[t]{3}{*}{ Age } & non R0 & 96 & 63.42 & 65.00 & 10.777 & 41 & 88 & 0.185 \\
\hline & Ro & 58 & 65.93 & 67.00 & 9.942 & 46 & 90 & \\
\hline & Total & 154 & 64.36 & 66.00 & 10.509 & 41 & 90 & \\
\hline \multirow[t]{3}{*}{ BMI } & non R0 & 96 & 27.466 & 26.600 & 5.6198 & 18.3 & 51.4 & 0.474 \\
\hline & Ro & 58 & 26.884 & 25.800 & 6.0783 & 15.4 & 58.0 & \\
\hline & Total & 154 & 27.247 & 26.400 & 5.7840 & 15.4 & 58.0 & \\
\hline \multirow[t]{3}{*}{ pre Tx CA125 } & non R0 & 92 & 2202.95 & 732.50 & 4335.145 & 27 & 28,000 & 0.530 \\
\hline & Ro & 57 & 1560.68 & 586.00 & 2284.097 & 40 & 11,100 & \\
\hline & Total & 149 & 1957.25 & 710.00 & 3691.556 & 27 & 28,000 & \\
\hline
\end{tabular}


Table 2 Absolute and relative frequencies for the categorical variables, by group and overall

\begin{tabular}{|c|c|c|c|c|c|}
\hline & Levels & Non RO & $\mathrm{RO}$ & Totals & $P$ \\
\hline RO category & & $51(57.3 \%)$ & $38(42.7 \%)$ & 89 & - \\
\hline \multirow[t]{7}{*}{ Charlson Comorbidy Index (CCI) } & 4 & $44(45.9 \%)$ & $32(55.2 \%)$ & $76(49.0 \%)$ & \multirow[t]{7}{*}{0.571} \\
\hline & 5 & $30(31.3 \%)$ & $12(20.7 \%)$ & $41(26.5 \%)$ & \\
\hline & 6 & $14(14.5 \%)$ & $9(15.5 \%)$ & $23(14.8 \%)$ & \\
\hline & 7 & $7(7.3 \%)$ & $3(5.2 \%)$ & $10(6.5 \%)$ & \\
\hline & 8 & $1(1.0 \%)$ & $1(1.7 \%)$ & $2(1.3 \%)$ & \\
\hline & 9 & - & $1(1.7 \%)$ & $1(0.6 \%)$ & \\
\hline & Totals & 96 & 58 & 153 & \\
\hline \multirow[t]{4}{*}{ Surgical Complexity Score (SCS) } & 0 & $54(56.3 \%)$ & $37(63.8 \%)$ & $91(58.7 \%)$ & \multirow[t]{4}{*}{0.333} \\
\hline & 1 & $30(31.3 \%)$ & $17(29.3 \%)$ & 47 (30.3\%) & \\
\hline & 2 & 12 & 4 & $16(10.3 \%)$ & \\
\hline & Totals & 96 & 58 & 154 & \\
\hline \multirow[t]{3}{*}{ Type of Surgery } & Primary & $17(17.7 \%)$ & $14(24.1 \%)$ & 31 (20.0\%) & \multirow[t]{3}{*}{0.451} \\
\hline & Interval & 79 (82.3\%) & 44 (75.9\%) & $123(80.0 \%)$ & \\
\hline & Totals & 96 & 58 & 154 & \\
\hline \multirow[t]{4}{*}{ Disease Score } & 1 & $14(14.6 \%)$ & $2(3.4 \%)$ & $16(10.3 \%)$ & \multirow[t]{4}{*}{0.0006} \\
\hline & 2 & 60 (62.5\%) & $28(48.3 \%)$ & 88 (56.8\%) & \\
\hline & 3 & 22 (22.9\%) & $28(48.3 \%)$ & 50 (32.3\%) & \\
\hline & Totals & 96 & 58 & 154 & \\
\hline
\end{tabular}

relevant to aEOC patients and their treating surgeons. The model uses available data items as input variables. These features can be readily available to the surgeons before performance of a laparotomy. Equally, it can be used at the research setting when reliable outcome data soon after surgery are required without long follow-up periods.
To predict CCR, our aim was to make predictions based on similar surgical outcomes from already-treated patients. The $k$-NN approach mirrors the way clinicians often talk about how they use their clinical experience to treat their patients.

Accurate prediction of CCR could allow identification of those patients, who, following primary chemotherapy,

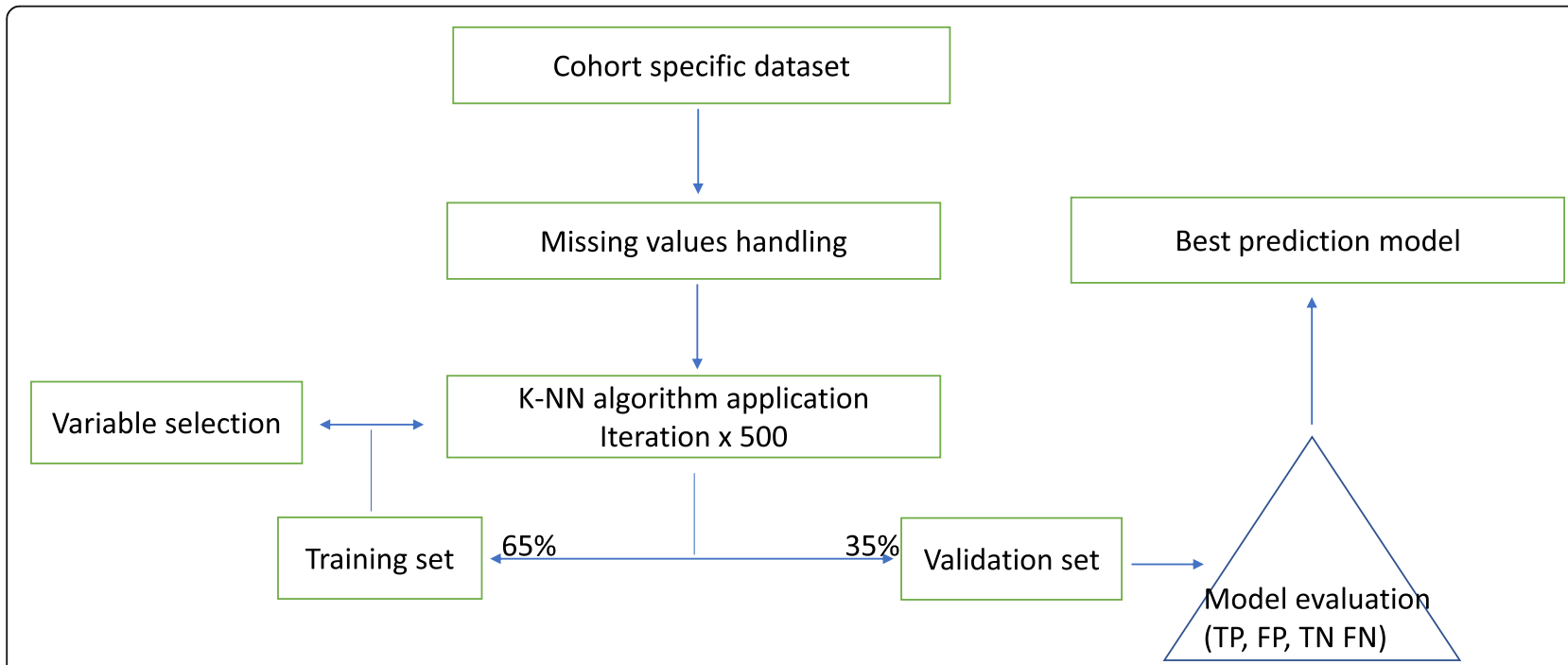

Fig. 1 K-NN modelling framework flowchart: The framework for building the predictive model comprised three steps: data pre-processing, model training and performance evaluation. TP: true positive, FP: false positive, TN: true negative, FN: false negative 
Table 3 Predictive accuracy of the k-NN model for different choices of the number of nearest neighbors $\times 500$ replications and comparison with conventional logistic regression. Accuracy selects the best number of neighbors within a larger range and uses predictor importance when calculating distances

\begin{tabular}{|c|c|c|c|c|c|c|c|}
\hline $\begin{array}{l}\text { Number of } \\
\text { nearest } \\
\text { neighbors }\end{array}$ & $\begin{array}{l}\text { Mean predictive } \\
\text { accuracy (\%) }\end{array}$ & $\begin{array}{l}\text { Minimum predictive } \\
\text { accuracy (\%) }\end{array}$ & $\begin{array}{l}\text { Maximum } \\
\text { predictive accuracy } \\
(\%)\end{array}$ & $\begin{array}{l}\text { Mean accuracy } \\
\text { of TPs (\%) }\end{array}$ & $\begin{array}{l}\text { Mean accuracy } \\
\text { of TNs (\%) }\end{array}$ & $\begin{array}{l}\text { Mean accuracy } \\
\text { of FPs (\%) }\end{array}$ & $\begin{array}{l}\text { Mean accuracy } \\
\text { of FNs (\%) }\end{array}$ \\
\hline 3 & 57.5 & 41.2 & 72.5 & 37.0 & 70.7 & 29.3 & 63.0 \\
\hline 4 & 57.8 & 39.2 & 78.4 & 38.2 & 70.6 & 29.4 & 61.8 \\
\hline 5 & 60.7 & 43.1 & 74.5 & 36.4 & 76.3 & 23.7 & 63.6 \\
\hline 6 & 60.8 & 41.2 & 82.4 & 37.5 & 75.8 & 24.2 & 62.5 \\
\hline 7 & 62.9 & 45.1 & 78.4 & 35.2 & 80.6 & 19.4 & 64.8 \\
\hline 8 & 62.8 & 43.1 & 78.4 & 35.7 & 80.2 & 19.8 & 64.3 \\
\hline 9 & 64.5 & 47.1 & 80.4 & 34.5 & 83.6 & 16.4 & 65.5 \\
\hline 10 & 64.4 & 47.1 & 78.4 & 35.0 & 83.2 & 16.8 & 65.0 \\
\hline 11 & 65.2 & 45.1 & 78.4 & 34.6 & 84.7 & 15.3 & 65.4 \\
\hline 12 & 64.7 & 43.1 & 82.4 & 34.8 & 83.9 & 16.1 & 65.2 \\
\hline 13 & 65.5 & 45.1 & 80.4 & 34.1 & 85.6 & 14.4 & 65.9 \\
\hline 14 & 65.3 & 45.1 & 80.4 & 33.7 & 85.5 & 14.5 & 66.3 \\
\hline 15 & 65.8 & 47.1 & 82.4 & 32.5 & 87.1 & 12.9 & 67.5 \\
\hline 16 & 65.4 & 47.1 & 80.4 & 32.0 & 86.8 & 13.2 & 68.0 \\
\hline 17 & 65.5 & 49.0 & 80.4 & 30.5 & 88.0 & 12.0 & 69.5 \\
\hline 18 & 65.5 & 47.1 & 76.5 & 29.8 & 88.3 & 11.7 & 70.2 \\
\hline 19 & 65.8 & 47.1 & 80.4 & 28.8 & 89.4 & 10.6 & 71.2 \\
\hline 20 & 65.6 & 43.1 & 80.4 & 28.1 & 89.5 & 10.5 & 71.9 \\
\hline $\begin{array}{l}\text { Logistic } \\
\text { regression } \\
\text { results }\end{array}$ & $\begin{array}{l}\text { Mean predictive } \\
\text { accuracy (\%) }\end{array}$ & $\begin{array}{l}\text { Minimum } \\
\text { predictive } \\
\text { accuracy (\%) }\end{array}$ & $\begin{array}{l}\text { Maximum } \\
\text { predictive } \\
\text { accuracy (\%) }\end{array}$ & $\begin{array}{l}\text { Mean } \\
\text { accuracy of } \\
\text { TPs (\%) }\end{array}$ & $\begin{array}{l}\text { Mean } \\
\text { accuracy of } \\
\text { TNs (\%) }\end{array}$ & $\begin{array}{l}\text { Mean } \\
\text { accuracy of } \\
\text { FPs (\%) }\end{array}$ & $\begin{array}{l}\text { Mean } \\
\text { accuracy of } \\
\text { FNs (\%) }\end{array}$ \\
\hline- & 63.4 & 2.0 & 80.4 & 42.7 & 76.7 & 23.1 & 57.1 \\
\hline
\end{tabular}

would not benefit from surgery and they should be considered for chemotherapy continuation. In that respect, the model would be useful for ongoing decision making and quality assurance during their cancer treatment pathway. Incomplete cytoreduction can be predicted with very high positive predictive value, close to $100 \%$ (i.e, if the test predicts residual disease regardless of the size, the R0 resection status will not be achieved) [18]. Nonetheless, a method to predict CCR with high likelihood (i.e. if the test predicts that no residual disease is achievable it will be achieved- the true negative) is still required. To serve this purpose, the major finding of this study is the prediction of CCR with a mean accuracy of true negatives as high as $90 \%$ for a tuning number of neighbors $k=20$ (Table 3).

The $k$-NN method is grounded in the idea that, to predict R0 resection following cytoreductive surgery, accuracy is more valid if one uses homogeneous subsamples. The more we know about a patient can potentially affect our surgical effort but, the more patients available, the more valuable the search for similar patients and the better the prediction of R0 resection. Such information can be particularly important to the elderly patients; as their cancer progresses or they experience increased comorbidities with age, often are too frail to be considered for cytoreductive surgery. Not infrequently, they question the effect of surgery on them or their subjective perception of pre-operative wellbeing is poor. A previous "good clinical experience" on surgical outcomes can be reassuring and may help refine risk stratification. Equally, pre-operative prediction of required surgery can focus on disease resectability rather than surgical complexity.

The main strength of the $k \mathrm{NN}$ approach is versatility. For instance, if the predictive method is planned to be used in a cancer screening system, specificity (false negatives) should be high; in a diagnostic system, both sensitivity (true positives) and specificity (true negatives) should be high. Testing the performance based on the number of nearest neighbors without choosing a classifier threshold allows us to keep all these applications feasible. In this way, many potential clinical applications should be captured by this model.

The mean predictive model accuracy was $66 \%$. Above $65 \%$ this is still satisfactory, but a number closer to $75 \%$ 


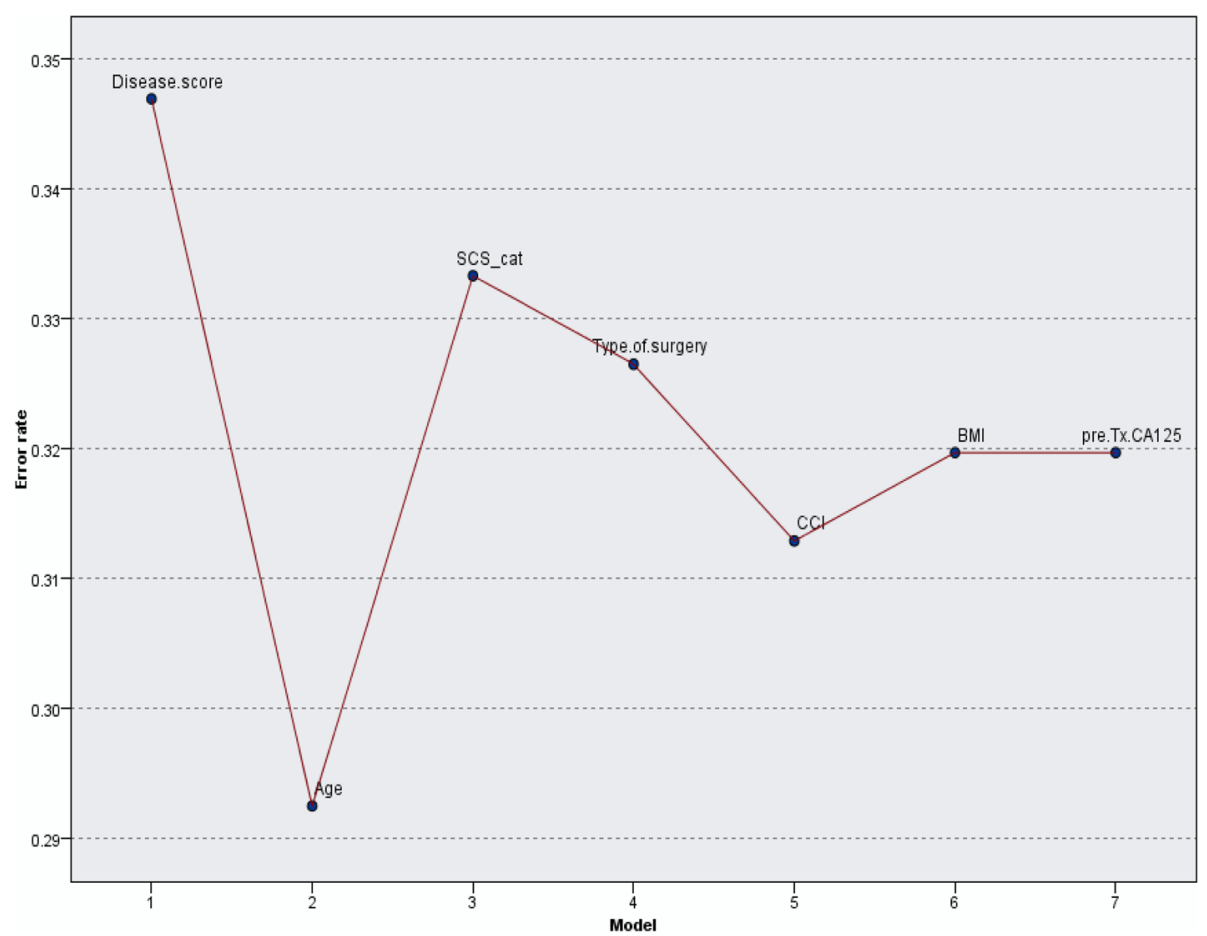

Fig. 2 Variable importance chart. Misclassification error for each predictor from the $k N N$ model for $k=15$. Selected predictors are weighted by their relative importance for $\mathrm{RO}$ resection prediction in the training set

Table 4 R0 resection prediction comparison between the kNN model and logistic regression on a random sub cohort of 20 patients from the training set. All these patients underwent primary or interval debulking surgery and complete cytoreduction was achieved

\begin{tabular}{|c|c|c|c|c|c|c|c|c|c|}
\hline Patient & Age & BMI & $\begin{array}{l}\text { Charlson Comorbidity } \\
\text { Index }\end{array}$ & $\begin{array}{l}\text { Type of } \\
\text { surgery }\end{array}$ & $\begin{array}{l}\text { Surgical Compexity Score } \\
\text { categories }\end{array}$ & $\begin{array}{l}\text { Pre Tx } \\
\text { Ca125 }\end{array}$ & $\begin{array}{l}\text { Disease } \\
\text { score }\end{array}$ & $\begin{array}{l}\text { kNN } \\
\text { model }\end{array}$ & $\begin{array}{l}\text { Logistic } \\
\text { regression }\end{array}$ \\
\hline 1 & 52 & 26.4 & 5 & Interval & Standard & 10,156 & 1 & Ro & Ro \\
\hline 2 & 66 & 28.1 & 5 & Interval & Standard & 466 & 1 & Ro & Ro \\
\hline 3 & 77 & 18.8 & 4 & Interval & Standard & 1876 & 2 & Ro & Ro \\
\hline 4 & 58 & 28.7 & 4 & Interval & Radical & 1900 & 2 & Ro & Ro \\
\hline 5 & 64 & 32.6 & 5 & Interval & Ultraradical & 1211 & 3 & Ro & Ro \\
\hline 6 & 51 & 23.8 & 4 & Interval & Radical & 1121 & 2 & Ro & Ro \\
\hline 7 & 52 & 39 & 4 & Interval & Standard & 586 & 2 & Ro & Ro \\
\hline 8 & 68 & 24.8 & 5 & Interval & Standard & 488 & 2 & Ro & Ro \\
\hline 9 & 48 & 31.9 & 5 & Interval & Standard & 3300 & 2 & RO & Ro \\
\hline 10 & 88 & 23 & 5 & Interval & Standard & 286 & 1 & Non-R0 & Ro \\
\hline 11 & 57 & 26.9 & 4 & Primary & Standard & 875 & 2 & Ro & Ro \\
\hline 12 & 57 & 38.8 & 5 & Interval & Radical & 1765 & 2 & Ro & Ro \\
\hline 13 & 69 & 28 & 4 & Interval & Standard & 1417 & 1 & Ro & Ro \\
\hline 14 & 68 & 28.7 & 6 & Interval & Standard & 52 & 2 & Non-RO & Non-R0 \\
\hline 15 & 50 & 33.9 & 4 & Interval & Standard & 2454 & 2 & Ro & Ro \\
\hline 16 & 55 & 33.2 & 4 & Interval & Standard & 1612 & 2 & Ro & Ro \\
\hline 17 & 68 & 21.6 & 4 & Interval & Ultraradical & 1800 & 2 & Ro & Ro \\
\hline 18 & 75 & 33.3 & 6 & Interval & Standard & 87 & 2 & Non-RO & Non-R0 \\
\hline 19 & 73 & 24.7 & 4 & Primary & Radical & 181 & 2 & Ro & Ro \\
\hline 20 & 57 & 19.5 & 4 & Primary & Ultraradical & 1176 & 2 & Ro & Ro \\
\hline
\end{tabular}


would have been preferable. One reason may be the fact that datasets based on clinical practice are often incomplete. If one variable value is missing, the entire case cannot be used in building the ML model. In our study, there was a very small however, amount of missing data. This limitation can be overcome by median imputation of the missing data. Another reason may be the high correlation amongst the variables that may render the model partly unstable due to collinearity (which further exists when the variables are increased). Use of fewer prediction variables would have rendered better accuracy at the cost of not collectively addressing the complexity of cytoreduction, leading to a less meaningful interpretation of outcome data. In the realm of precision medicine, the ability of ML models to discover embedded patterns within data by handling multiple factors at once, irrespective of the data size may lead to a better understanding of the complexity in achieving CCR. Cohort expansion to a larger sample size is expected to improve predictability. Prospective internal validation of the model to a larger cohort is currently ongoing.

Our results also suggest that the $k$-NN predictions of CCR slightly outperform those predicted by conventional statistics. That is, the strategy where predictions are based on small subsamples of patients with similar clinical characteristics appears superior to the strategy of basing predictions on optimally weighted combinations of clinical characteristics. This is likely due to extraction of homogeneous samples with high similarity to any individual patient. The study sample was not large but offered enough similar neighbors on average to allow for accurate predictions. Use of $k-\mathrm{NN}$ as a tuning parameter gives non inferior results compared to multiple logistic regressions, indicating that $\mathrm{R} 0$ prediction in aEOC can be made stable irrespective of prediction models.

From the variables tested to contribute in $\mathrm{R} 0$ prediction, only disease score was statistically significant. Nevertheless, this would not affect $k$-NN model accuracy; it is the relative importance of each predictor in estimating the model. Selected predictors were weighted equally against each other in selecting $\mathrm{NNs}$ and may have played a role in the success in predicting the surgical outcome. Some variables may be differentially weighted in the $\mathrm{NN}$ analysis to reflect estimates of their contribution to relevant similarity (Fig. 2). Variable importance does not necessarily relate to model accuracy. It relates to the importance of each variable in making a prediction, not whether the prediction is accurate.

Other studies used an ordinal classification method to predict surgical outcomes in aEOC patients with a $64.9 \%$ accuracy and AUC of 0.697 (R0 vs non-R0) based solely on preoperative information [19]. Another AI model predicted the outcome of surgery and again showed that ANN could predict outcome (optimal cytoreduction vs. suboptimal cytoreduction) with $77 \%$ accuracy and an AUC of 0.73. Application of AI weighted the importance of factors predicting $\mathrm{CCR}$ at secondary cytoreductive surgery for recurrent ovarian cancer [20].

This retrospective study was a single institution experience with limited heterogeneity in the study population, which may differ from other tertiary unit settings. It was not powered to test how many overall cases are necessary to define sufficiently homogeneous subgroups of NNs, which are adequate for predictions. Nevertheless, ML retains the strength of the structural model used for the R0 prediction even when applied in other populations and reveal different prediction features.

\section{Conclusions}

We considered the problem of predicting CCR in aEOC patients using clinical preoperative and intraoperative variables and focused our analysis on the comparison between $\mathrm{AI}$ and conventional regression models under the same resampling conditions. The study demonstrated the feasibility of using the $k$-NN approach, which is very much reflective of "previous clinical experience" for accurate prediction of $\mathrm{R} 0$ resection during aEOC surgery. The model slightly outperforms conventional logistic regression. It should be further improved with data expansion and become directly available to clinicians.

\section{Acknowledgements}

We would like to thank all the members of the Gynaecological Oncology team at St James's University Hospital, Leeds who managed the patients enrolled in the underlying study.

\section{Authors' contributions}

Alexandros Laios: Conceptualisation, data collection and analysis, writing draft, review and editing. Alexandros Gryparis: Statistical analysis and writing. Diederick DeJong: Data collection, review and editing. Richard Hutson: Review and editing. Giorgios Theophilou: Conceptualisation, review and editing. Chris Leach: Conceptualisation, statistical advice, writing, review and editing. The author(s) read and approved the final manuscript.

Funding

There was no funding required for this study.

Availability of data and materials

The data that support the findings of this study are available from the corresponding author upon reasonable request.

Ethics approval and consent to participate

The present study was conducted in accordance with the Declaration of Helsinki. The study protocol was approved by the Research Ethics Committee Leeds Teaching Hospitals (ID 282396) with a waiver of informed consent.

\section{Consent for publication}

No consent was required.

Competing interests

None of the authors declared any conflict of interest regarding the subject of the study.

\section{Author details}

${ }^{1}$ Department of Gynaecological Oncology, St James's University Hospital, Leeds Teaching Hospitals, Leeds LS9 7TF, UK. ${ }^{2}$ Unit of Endocrinology, 
Diabetes Mellitus and Metabolism, Aretaion Hospital, National and Kapodistrian University of Athens School of Medicine, Athens, Greece. ${ }^{3}$ School of Human \& Health Sciences, University of Huddersfield, Huddersfield HD1 3DH, UK. ${ }^{4}$ Department of Psychology Services, South West Yorkshire Mental Health NHS Foundation Trust, The Laura Mitchell Health \& Wellbeing Centre, Halifax HX1 1YR, UK.

Received: 12 May 2020 Accepted: 4 August 2020

Published online: 29 September 2020

\section{References}

1. UK CR. Statistics and outlook for ovarian cancer2016.

2. Querleu D, Planchamp F, Chiva L, Fotopoulou C, Barton D, Cibula D, et al. European Society of Gynaecological Oncology (ESGO) guidelines for ovarian Cancer surgery. Int J Gynecol Cancer. 2017;27(7):1534-42. https://doi.org/10. 1097/IGC.0000000000001041.

3. Elattar A, Bryant A, Winter-Roach BA, Hatem M, Naik R. Optimal primary surgical treatment for advanced epithelial ovarian cancer. Cochrane Database Syst Rev. 2011;2011(8):Cd007565. https://doi.org/10.1002/ 14651858.CD007565.pub2.

4. Wright AA, Bohlke K, Armstrong DK, Bookman MA, Cliby WA, Coleman RL, et al. Neoadjuvant chemotherapy for newly diagnosed, advanced ovarian Cancer: Society of Gynecologic Oncology and American Society of clinical oncology clinical practice guideline. J Clin Oncol. 2016;34(28):3460-73. https://doi.org/10.1200/JCO.2016.68.6907.

5. Peiretti M, Zanagnolo V, Aletti GD, Bocciolone L, Colombo N, Landoni F, et al. Role of maximal primary cytoreductive surgery in patients with advanced epithelial ovarian and tubal cancer: surgical and oncological outcomes. Single institution experience. Gynecol Oncol. 2010;119(2):259-64. https://doi.org/10.1016/j.ygyno.2010.07.032.

6. Kourou K, Exarchos TP, Exarchos KP, Karamouzis MV, Fotiadis DI. Machine learning applications in cancer prognosis and prediction. Comput Struct Biotechnol J. 2015;13:8-17. https://doi.org/10.1016/j.csbj.2014.11.005 eCollection 2015.

7. Kadhel P, Revaux A, Carbonnel M, Naoura I, Asmar J, Ayoubi JM. An update on preoperative assessment of the resectability of advanced ovarian cancer. Horm Mol Biol Clin Invest. 2019;/j/hmbci.ahead-of-print/hmbci-2019-0032/ hmbci-2019-0032.xml;019. https://doi.org/10.1515/hmbci-2019-0032.

8. Nagarajan N, Yapp EKY, Le NOK, Kamaraj B, Al-Subaie AM, Yeh HY Application of Computational Biology and Artificial Intelligence Technologies in Cancer Precision Drug Discovery. Biomed Res Int. 2019; 2019:8427042. Published 2019 Nov 11. https://doi.org/10.1155/2019/ 8427042 .

9. Huang C, Mezencev R, McDonald JF, Vannberg F. Open source machine learning algorithms for the prediction of optimal cancer drug therapies. PLoS One. 2017;12(10):e0186906. Published 2017 Oct 26. https://doi.org/10. 1371/journal.pone.0186906

10. Gupta S, Tran T, Luo W, Phung D, Kennedy RL, Broad A, et al. Machinelearning prediction of cancer survival: a retrospective study using electronic administrative records and a cancer registry. BMJ Open. 2014;4(3):e004007. https://doi.org/10.1136/bmjopen-2013-004007.

11. Jefferson MF, Pendleton N, Lucas SB, Horan MA. Comparison of a genetic algorithm neural network with logistic regression for predicting outcome after surgery for patients with nonsmall cell lung carcinoma. Cancer. 1997; 79(7):1338-42. https://doi.org/10.1002/(sici)1097-0142(19970401)79:7<1338:: aid-cncr10>3.0.co;2-0.

12. Brabec $B$, Meister R. A nearest-neighbor model for regional avalanche forecasting. Ann Glaciol. 2001;32:130-4.

13. Lutz W, Leach C, Barkham M, Lucock M, Stiles WB, Evans C, et al. Predicting change for individual psychotherapy clients on the basis of their nearest neighbors. J Consult Clin Psychol. 2005;73(5):904-13. https://doi.org/10. 1037/0022-006X.73.5.904

14. Hastie T, Friedman JH. The elements of statistical learning: Data mining, inference, and prediction. 2nd ed. New York: Springer; 2016.

15. Team RC. R: a language and environment for statistical computing. Vienna: R Foundation for Statistical Computing; 2016.

16. IBM SPSS Statistics for Windows. Version 25.0. Armonk: IBM Corp: 2017.

17. du Bois A, Reuss A, Pujade-Lauraine E, Harter P, Ray-Coquard I, Pfisterer J. Role of surgical outcome as prognostic factor in advanced epithelial ovarian cancer: a combined exploratory analysis of 3 prospectively randomized phase 3 multicenter trials. Cancer. 2009;115(6):1234-44. https://doi.org/10. 1002/cncr.24149.

18. Fagotti A, Ferrandina G, Fanfani F, Garganese G, Vizzielli G, Salerno MG, et al. Prospective validation of a laparoscopic predictive model for optimal cytoreduction in advanced ovarian carcinoma. Am J Obstet Gynecol. 2008; 199(6):642.e641-6. https://doi.org/10.1016/j.ajog.2008.06.052.

19. Kawakami E, Tabata J, Yanaihara N, Ishikawa T, Koseki K, lida Y, et al. Application of artificial intelligence for preoperative diagnostic and prognostic prediction in epithelial ovarian Cancer based on blood biomarkers. Clin Cancer Res. 2019;25(10):3006-15. https://doi.org/10.1158/ 1078-0432.CCR-18-3378.

20. Bogani G, Rossetti D, Ditto A, Martinelli F, Chiappa V, Chiappa L, et al. Artificial intelligence weights the importance of factors predicting complete cytoreduction at secondary cytoreductive surgery for recurrent ovarian cancer. J Gynecol Oncol. 2018;29(5):e66. https://doi.org/10.3802/jgo.2018.29. e66.

\section{Publisher's Note}

Springer Nature remains neutral with regard to jurisdictional claims in published maps and institutional affiliations.
Ready to submit your research? Choose BMC and benefit from:

- fast, convenient online submission

- thorough peer review by experienced researchers in your field

- rapid publication on acceptance

- support for research data, including large and complex data types

- gold Open Access which fosters wider collaboration and increased citations

- maximum visibility for your research: over $100 \mathrm{M}$ website views per year

At $\mathrm{BMC}$, research is always in progress.

Learn more biomedcentral.com/submissions 